International Journal of Pure and Applied Mathematics

Volume 105 No. 4 2015, 639-645

ISSN: 1311-8080 (printed version); ISSN: 1314-3395 (on-line version)

url: http://www.ijpam.eu

doi: http://dx.doi.org/10.12732/ijpam.v105i4.5

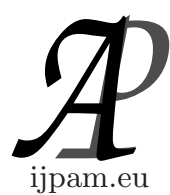

\title{
COLORABILITY OF UNITARY CAYLEY DIGRAPHS OF CYCLIC GROUPS
}

\author{
C. Promsakon \\ Department of Mathematics \\ Faculty of Applied Science \\ King Mongkut's University of Technology North Bangkok \\ Bangkok, 10800, THAILAND
}

\begin{abstract}
Let $n$ be a positive integer greater than $1, \mathbb{Z}_{n}$ the integer modulo $n$ and $A$ the subset of $\mathbb{Z}_{n}$. The Cayley digraph of $\mathbb{Z}_{n}$ with a connecting set $A$, denoted by $\operatorname{Cay}\left(\mathbb{Z}_{n}, A\right)$ is a digraph whose vertex set is $\mathbb{Z}_{n}$ and a vertices $x$ and $y$ are adjacent if and only if $y=x+a$ where $a \in A$. We call a Cayley digraph as unitary Cayley digraph if its connecting set is the set of all units in $\mathbb{Z}_{n}$.

In this paper, we focus on coloring properties of unitary Cayley digraphs of $\mathbb{Z}_{n}$. We show their chromatic numbers and their edge chromatic number.
\end{abstract}

AMS Subject Classification: 46MO5, 05C40, 05C76

Key Words: Cayley graphs, coloring, chromatic number, edge-chromatic number, units

\section{Introduction}

Let $S$ be a semigroup, and let $A$ be a nonempty subset of $S$. The Cayley

Received: June 19, 2015

(c) 2015 Academic Publications, Ltd. url: www.acadpubl.eu 
digraph $\operatorname{Cay}(S, A)$ of $S$ with a connecting set $A$ is defined as the digraph with a vertex set $S$ and an edge set $E(S)$ consisting of ordered pairs $(x, x a)$ with $x \in S$ and $a \in A$.

The Cayley digraphs play a significant role in digraph theory because they encodes a semigroup structure into a digraph structure. There are many properties of Cayley digraphs that were explored. One of them is for a model of interconnection networks, such as De Bruijin and Kautz digraphs. The Cayley digraphs that we focus are Cayley digraph of the integer modulo $n$ with a connecting set $U_{n}$, the set of all units in $\mathbb{Z}_{n}$.

For example, We consider $\operatorname{Cay}\left(\mathbb{Z}_{6}, U_{6}\right)$. We have $U_{6}=\{1,5\}$ and a graph of $\operatorname{Cay}\left(\mathbb{Z}_{6}, U_{6}\right)$ is a cycle of length 6 showed in a below figure.

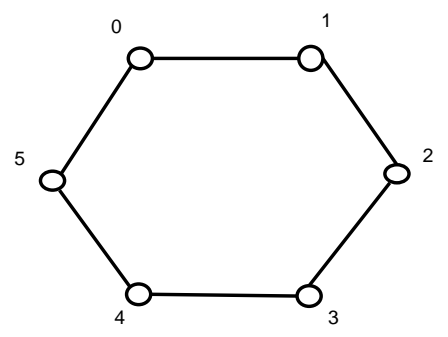

Figure 1: A digraph $\operatorname{Cay}\left(\mathbb{Z}_{6}, U_{6}\right)$

The main objectives of this work are to find the chromatic numbers and the edge chromatic numbers of unitary Cayley digraphs of integer modulo $n$. Some characterisations are investigated too. We start by give definitions and properties about graph coloring.

A $k$-coloring of a graph $G$ is a labeling of vertices in $G, f: V(G) \rightarrow$ $\{1,2, \ldots, k\}$. We call the image of a coloring as colors. A $k$-coloring is called proper if all adjacent vertices in $G$ have different labels. A graph is called $k$ colorable if it has a proper $k$-coloring. We always use a coloring for a proper coloring. The chromatic number of a graph $G$, denoted by $\chi(G)$, is the least $k$ such that $G$ is $k$-colorable.

Theorem 1 ([3]). A graph $G$ is a bipartite graph if and only if $\chi(G)=2$.

A clique of a graph $G$ is a complete subgraph of $G$. The clique number of a graph $G$, denoted by $\omega(G)$, is the maximum order of cliques of $G$. Clearly that for each vertices in a complete graph, they are adjacent. So they have different labels in a coloring. Hence $\chi\left(K_{n}\right)=n=\omega\left(K_{n}\right)$.

Proposition $2([3])$. For any graph $G, \chi(G) \geq \omega(G)$. 
Theorem 3 (Seinsehe). If a graph $G$ has no induced subgraph isomorphic to $P_{4}$, then $\chi(G)=\omega(G)$.

The greedy algorithm is an algorithm to find the chromatic number of any graphs. The greedy algorithm takes an order of vertices $v_{1}, v_{2}, \ldots, v_{n}$ and colors vertices in that order, assigning to each vertex the smallest indexed color not already use by it previously colored neighbors. By the greedy algorithm, we have a bound of the chromatic number of a graph.

Theorem 4 ([3]). For any graph $G, \chi(G) \leq \Delta(G)+1$.

Clearly that an equality in Theorem 4 holds in two cases which are odd cycles and complete graphs. Next, R. Leonard Brooks proved that these are the only connected graphs for which the bound is attained.

Theorem 5 (Brooks's Theorem). For every connected graph $G$ that is not an odd cycle or a complete graph, $\chi(G) \leq \Delta(G)$.

The edge chromatic number of a graph $G$, denoted as $\chi^{\prime}(G)$, is defined similarly to the chromatic number that is fewest number of colors necessary to color each edge of $G$ such that no two edges incident on the same vertex have the same color. In other words, it is the number of distinct colors in a minimum edge coloring. Vizing's theorem (named for Vadim G. Vizing who published it in 1964) states bound is almost tight as following theorem

Theorem 6. For any simple graph $G$, we have $\Delta(G) \leq \chi^{\prime}(G) \leq \Delta(G)+1$.

For any simple graph $G$, the edge chromatic number of $G$ is either $\Delta(G)$ or $\Delta(G)+1$. When $\chi^{\prime}(G)=\Delta(G), G$ is said to be of class 1 , otherwise, it is said to be of class 2 .

The next section, we focus on colorability of unitary Cayley digraphs and some their properties.

\section{The Chromatic Numbers of Unitary Cayley Digraphs of $\mathbb{Z}_{n}$}

This section we introduce an unitary Cayley digraphs of $\mathbb{Z}_{n}$. We start by giving definitions of units and unitary Cayley digraphs of $\mathbb{Z}_{n}$.

For any positive integer $n$, an unit of $\mathbb{Z}_{n}$ is an element in $\mathbb{Z}_{n}$ such that have an inverse under multiplication. We denote $U_{n}$ as a set of all units in $\mathbb{Z}_{n}$. It is well-known that $a$ is an unit in $\mathbb{Z}_{n}$ if and only if $\operatorname{gcd}(a, n)=1$ and the number of all units in $\mathbb{Z}_{n}$ is equal to $\phi(n)$, the Euler-phi function. Hence $\left|U_{n}\right|=\phi(n)$. Moreover, $U_{n}$ is a group under multiplication. 
Lemma 7. Let $n$ be any positive integer. If $p$ is the smallest prime factor of $n$, then $U_{p}$ is a subset of $U_{n}$.

Proof. Assume that $p$ is the smallest factor of $n$. Let $x$ be an element in $U_{p}$. Then $1 \leq x \leq p-1$. Since $p$ is the smallest prime factor, we have for any prime factor of $n$ does not divide $x$. Hence $\operatorname{gcd}(x, n)=1$ and $x \in U_{n}$.

Let $n$ be a positive integer greatten than $1, \mathbb{Z}_{n}$ the integer modulo $n$ and $A$ the subset of $\mathbb{Z}_{n}$. The Cayley digraph of $\mathbb{Z}_{n}$ with a connecting set $A$, denoted by $\operatorname{Cay}\left(\mathbb{Z}_{n}, A\right)$ is a digraph whose vertex set is $\mathbb{Z}_{n}$ and a vertices $x$ and $y$ are adjacent if and only if $y-x \in A$. In case that a connecting set $A$ is a set of all units $U_{n}$, we call that Cayley digraph as a Unitary Cayley digraphs denote as $\operatorname{Cay}\left(\mathbb{Z}_{n}, U_{n}\right)$.

Now we will show that for any postiive integer $n, \operatorname{Cay}\left(\mathbb{Z}_{n}, U_{n}\right)$ is an undirected graph which mean if a vertex $u$ is adjacent to a vertex $v$, then $v$ is also adjacent to $u$.

Theorem 8. Let $n$ be a natural number exceed than 1 and $U_{n}$ be a set of all units in $\mathbb{Z}_{n}$. A digraph $\operatorname{Cay}\left(\mathbb{Z}_{n}, U_{n}\right)$ is a loopless undirected graph.

Proof. It is clearly that for any $u \in \mathbb{Z}_{n}, u-u=0 \notin U_{n}$. Hence there is no loop at vertex $u$ and $\operatorname{Cay}\left(\mathbb{Z}_{n}, U_{n}\right)$ has no loop. Next we show that $\operatorname{Cay}\left(\mathbb{Z}_{n}, U_{n}\right)$ is undirected. Let $u$ and $v$ be vertices of $\operatorname{Cay}\left(\mathbb{Z}_{n}, U_{p}\right)$ such that $u$ is adjacent to $v$. Then $v-u \in U_{n}$. So $\operatorname{gcd}(u-v, n)=\operatorname{gcd}(v-u, n)=1$ and $u-v \in U_{n}$. Therefore $v$ is adjacent to $u$ and $\operatorname{Cay}\left(\mathbb{Z}_{n}, U_{n}\right)$ is undirected.

From now on, we will call unitary Cayley digraph as unitary Cayley graph because it is undirected. We show next some properties and some characterisation of unitary Cayley graphs.

Theorem 9. Let $n$ be any positive integer and $p$ the smallest prime factor of $n$. Then $\operatorname{Cay}\left(\mathbb{Z}_{p}, U_{p}\right)$ is a subgraph of $\operatorname{Cay}\left(\mathbb{Z}_{n}, U_{n}\right)$.

Proof. By Lemma 7, we know that $U_{p}$ is a subset of $U_{n}$. So $\operatorname{Cay}\left(\mathbb{Z}_{n}, U_{p}\right)$ is a subgraph of $\operatorname{Cay}\left(\mathbb{Z}_{n}, U_{n}\right)$. Since $\operatorname{Cay}\left(\mathbb{Z}_{p}, U_{p}\right)$ is a subgraph of $\operatorname{Cay}\left(\mathbb{Z}_{n}, U_{p}\right)$, we can conclude that $\operatorname{Cay}\left(\mathbb{Z}_{p}, U_{p}\right)$ is a subgraph of $\operatorname{Cay}\left(\mathbb{Z}_{n}, U_{n}\right)$.

We will show that $\operatorname{Cay}\left(\mathbb{Z}_{2 n}, U_{2 n}\right)$ is a bipartite graph and its chromatic number is 2 in the next theorem.

Theorem 10. For any integer $n, \chi\left(\operatorname{Cay}\left(\mathbb{Z}_{2 n}, U_{2 n}\right)\right)=2$ and $\operatorname{Cay}\left(\mathbb{Z}_{2 n}, U_{2 n}\right)$ is a bipartite graph. 
Proof. Let $n$ be a positive integer. Define $f: \mathbb{Z}_{2 n} \rightarrow\{1,2\}$ by

$$
f(n)=\left\{\begin{array}{ll}
0 & \text { if } n \equiv 0 \\
1 & \text { if } n \equiv 1
\end{array} \quad(\bmod 2)\right.
$$

Let $u$ and $v$ be elements in $\mathbb{Z}_{2 n}$ such that $u$ and $v$ are adjacent in $\operatorname{Cay}\left(\mathbb{Z}_{2 n}, U_{2 n}\right)$. Then $u+v \in U_{2 n}$ and hence $u+v \equiv 1(\bmod 2)$. So $u \not \equiv v(\bmod 2)$ and then $f(u) \neq f(v)$. It is clearly that there is an edge in $\operatorname{Cay}\left(\mathbb{Z}_{2 n}, U_{2 n}\right)$. Therefore $\chi\left(\operatorname{Cay}\left(\mathbb{Z}_{2 n}, U_{2 n}\right)\right)=2$ and $\operatorname{Cay}\left(\mathbb{Z}_{2 n}, U_{2 n}\right)$ is a bipartite graph.

We give some trivial facts about $\operatorname{Cay}\left(\mathbb{Z}_{n}, U_{n}\right)$ that we use in this work. It is easy to see that each vertex in $\operatorname{Cay}\left(\mathbb{Z}_{n}, U_{n}\right)$ has degree $\left|U_{n}\right|=\phi(n)$. Hence $\operatorname{Cay}\left(\mathbb{Z}_{n}, U_{n}\right)$ is $\phi(n)$ regular graph. Next, we show conditions for $n$ to make $\operatorname{Cay}\left(\mathbb{Z}_{n}, U_{n}\right)$ be odd cycle and complete graph.

Theorem 11. Let $n$ be a positive integer, $U_{n}$ a set of all units in $\mathbb{Z}_{n}$. A graph $\operatorname{Cay}\left(\mathbb{Z}_{n}, U_{n}\right)$ is an odd cycle if and only if $n=3$.

Proof. It is clearly that $\operatorname{Cay}\left(\mathbb{Z}_{3}, U_{3}\right)$ is a odd cycle. Suppose that $\operatorname{Cay}\left(\mathbb{Z}_{n}, U_{n}\right)$ is a odd cycle. Then $n$ is odd and $\left|U_{n}\right|=\phi(n)=2$. If $n=a b$ where $a \neq 1$, $b \neq 1$ and $(a, b)=1$, then both $a$ and $b$ are odd and $\phi(n)=\phi(a) \phi(b)>2$. Hence $n$ is a prime number,. Therefore $n=3$.

Next, we give a charecter of $\operatorname{Cay}\left(\mathbb{Z}_{p}, U_{p}\right)$ where $p$ is a prime number. For any prime number $p$, we have $U_{p}=\mathbb{Z}_{p} \backslash\{0\}$. So $\operatorname{deg}(u)=p-1$ for all vertex $u$ in $\mathbb{Z}_{p}$. Since $\operatorname{Cay}\left(\mathbb{Z}_{p}, U_{p}\right)$ is loopless, we can conclude that $\operatorname{Cay}\left(\mathbb{Z}_{p}, U_{p}\right)$ is a complete graph.

Theorem 12. Let $n$ be a positive integer. Then $\operatorname{Cay}\left(\mathbb{Z}_{n}, U_{n}\right)$ is a complete graph if and only if $n$ is a prime number.

Proof. As we mention above, we conclude that if $n$ is a prime number, then $\operatorname{Cay}\left(\mathbb{Z}_{n}, U_{n}\right)$ is a complete graph. Suppose $n$ is not prime. Then $\left|U_{n}\right|=\phi(n)<$ $n-1$ and $\Delta\left(\operatorname{Cay}\left(\mathbb{Z}_{n}, U_{n}\right)\right)<n-1$. Therefore $\operatorname{Cay}\left(\mathbb{Z}_{n}, U_{n}\right)$ is not a complete graph.

Theorem 13. Let $n$ be any positive integer and $p$ the smallest prime factor of $n$. Then $\chi\left(\operatorname{Cay}\left(\mathbb{Z}_{n}, U_{n}\right)\right) \geq p$

Proof. By Theorem 9 and Theorem 12, we conclude that $\chi\left(\operatorname{Cay}\left(\mathbb{Z}_{n}, U_{n}\right)\right) \geq$ $\chi\left(\operatorname{Cay}\left(\mathbb{Z}_{p}, U_{p}\right)\right)=p$ 
By applying Brooks's Theorem, we have a bound of the chromatic number of Cayley graph of $\mathbb{Z}_{n}$ as follow.

Theorem 14. Let $n$ be a positive integer. If $n$ is a prime number, then $\chi\left(\operatorname{Cay}\left(\mathbb{Z}_{n}, U_{n}\right)\right)=n$. Otherwise $\chi\left(\operatorname{Cay}\left(\mathbb{Z}_{n}, U_{n}\right)\right) \leq \phi(n)$.

The upper bound in Theorem 14 is too loose. For example, We know that $\operatorname{Cay}\left(\mathbb{Z}_{2 n}, U_{2 n}\right)$ is a bipartite graph. So $\chi\left(\operatorname{Cay}\left(\mathbb{Z}_{2 n}, U_{2 n}\right)=2\right.$ while $\phi(2 n)=$ $\phi(n)>2$.

Lemma 15. Let $n$ be any positive integer and $p$ the smallest prime factor of $n$. A set $A_{i}=\left\{x \in \mathbb{Z}_{n} \mid x \equiv i(\bmod p)\right\}$ is a independent set of $\operatorname{Cay}\left(\mathbb{Z}_{n}, U_{n}\right)$ for any $i \in\{0,1,2, \ldots p-1\}$.

Proof. Let $i \in\{0,1,2, \ldots p-1\}$. Assume $x$ and $y$ are elements in $A_{i}=\{x \in$ $\left.\mathbb{Z}_{n} \mid x \equiv i(\bmod p)\right\}$. So $x \equiv i \equiv y(\bmod p)$ and then $x-y \equiv 0(\bmod p)$. Hence $\operatorname{gcd}(x-y, n) \neq 1$. That implies $x-y \notin U_{n}$. Therefore $x$ is not adjacent to $y$ in $\operatorname{Cay}\left(\mathbb{Z}_{n}, U_{n}\right)$ and $A_{i}=\left\{x \in \mathbb{Z}_{n} \mid x \equiv i(\bmod p)\right\}$ is a independent set.

As we known in Lemma 15, there are $p$ distinct sets of independent sets. If we give distinct color to each independent set, we have the the chromatic number of unitary Cayley graphs is at most $p$. By applying Theorem 13 and Lemma 15, we can conclude the chromatic numbers of $\operatorname{Cay}\left(\mathbb{Z}_{n}, U_{n}\right)$ as the following theorem.

Theorem 16. Let $n$ be any positive integer and $p$ the smallest prime factor of $n$. The chromatic number of $\operatorname{Cay}\left(\mathbb{Z}_{n}, U_{n}\right)$ is $p$ (a clique number)

By Theorem 16, we conclude that the chromatic numbers of unitary Cayley graphs are their clique numbers. Next, we show that unitary Cayley graphs are in class 1 , the edge chromatic number are their maximum degree.

Theorem 17. Let $n$ be any positive integer. The edge chromatic number of unitary Cayley graph $\operatorname{Cay}\left(\mathbb{Z}_{n}, U_{n}\right)$ is its maximum degree.

Proof. We set $\mathbb{Z}_{n}=\{1,2,3, \ldots, n\}$ and $U_{n}=\left\{u_{1}, u_{2}, u_{3}, \ldots, u_{\phi(n)}\right\}$. Define $f: E\left(\operatorname{Cay}\left(\mathbb{Z}_{n}, U_{n}\right) \rightarrow U_{n}\right.$ by $f(x y)=u_{i}$ if $x-y \equiv u_{i}(\bmod n)$. Suppose $x, y$ and $z$ are distinct elements in $\mathbb{Z}_{n}$ such that $x y, y z \in E\left(\operatorname{Cay}\left(\mathbb{Z}_{n}, U_{n}\right)\right.$. Then $(x-y)-(y-z)=x-z \not \equiv 0(\bmod n)$ and so $f(x y)=x-y \not \equiv y-z=f(y z)$. Hence $f(x y) \neq f(y z)$ and $\chi^{\prime}\left(\operatorname{Cay}\left(\mathbb{Z}_{n}, U_{n}\right) \leq\left|U_{n}\right|=\phi(n)=\Delta\left(\operatorname{Cay}\left(\mathbb{Z}_{n}, U_{n}\right)\right)\right.$. Because $\chi^{\prime}\left(\operatorname{Cay}\left(\mathbb{Z}_{n}, U_{n}\right) \geq \Delta\left(\operatorname{Cay}\left(\mathbb{Z}_{n}, U_{n}\right)\right.\right.$, we conclude that $\chi^{\prime}\left(\operatorname{Cay}\left(\mathbb{Z}_{n}, U_{n}\right)=\right.$ $\Delta\left(\operatorname{Cay}\left(\mathbb{Z}_{n}, U_{n}\right)\right.$. 


\section{Acknowledgments}

This research was funded by the Faculty of Applied Sciences, King Mongkuls University of Technology North Bangkok, Thailand, Project No. 5842103.

\section{References}

[1] G. Chartrand and P. Zhang, Introduction to graph theory, McGraw-Hill Companies, Inc., 2005.

[2] L Collins, Ann Trenk, Nordhaus-Gaddum theorem for the dis- tinguishing chromatic number, The electronic journal of combina- torics 16 (2009), arXiv:1203.5765v1 [math.CO], 26 March 2012.

[3] B. W. Douglas, Introduction to graph theory, Prentice-Hall Inc., 2001.

[4] D. S. Dummit and R. M. Foote, Abstract Algebra, Hoboken, NJ:Wiley, 2004.

[5] T. W. Hungerford, Algebra,Springer-Verlag New York, Inc., 1974.

[6] A. V. Kelarev, On undirected Cayley graphs, Australasian Journal of Combinatorics. 25 (2002), 73-78.

[7] C. Promsakon, Undirectedness, connectedness and chromatic number of endo-Cayley digraphs of some semigroups, Ph.D. Thesis, Chiang Mai Universitat, Chiang Mai, 2013.

[8] C. Promsakon and S. Panma, Undirected endo-Cayley digraphs of cyclic groups of order primes, International science index., 8(2014), 1958-1962.

[9] V. G. Vizing, "On an Estimate of the Chromatic Class of a p-Graph" [Russian]. Diskret. Analiz, 3(1964), 23-30. 
